\title{
Emerging Group B Streptococcus Infection
}

\author{
Arielle Turpin ${ }^{\mathrm{a}, \mathrm{b}}$, Ana Mendes ${ }^{\mathrm{a}}$, Diogo Ribeiro ${ }^{\mathrm{a}}$, Victor Fernandes ${ }^{\mathrm{a}}$, \\ Duarte Garcao ${ }^{\text {a }}$, Manuel Caneira ${ }^{\text {a }}$, Hugo Freitas ${ }^{\mathrm{a}}$, \\ Miguel Neves ${ }^{\mathrm{a}}$, Fatima Xambre ${ }^{\mathrm{a}}$
}

\begin{abstract}
Necrotizing fasciitis (NF) from Streptococcus agalactiae (GBS) and toxic shock-like syndrome (TSLS) are rare entities that have yet to be described in a burn patient. We report the case of a patient with $72 \%$ total body surface area burn that simultaneously developed GBS NF and TSLS. Aggressive measures were required for complete recovery.
\end{abstract}

Keywords: Necrotizing fasciitis; Group B streptococcus; Toxic shock-like syndrome; Burn

\section{Introduction}

Necrotizing fasciitis (NF) is a severe form of infection of fascia and subcutaneous tissue that rapidly tracks through the fascial plane. It has a high rate of mortality that reaches $36 \%$ in some series [1]. It is usually initiated by a breach in cutaneous lining and less frequently from mucosal or hematogenous dissemination. It can be associated with systemic toxicity caused by superantigens of staphylococcal or streptococcal origin.

While group A Streptococcus pyogenes (GAS) is the most frequent agent of monomicrobial NF, group B Streptococcus agalactiae (GBS) is an emerging one with only few more than 20 cases described in English-based scientific literature [2, 3]. It's postulated, although not proven, that GAS virulence factors were transfected to GBS giving it added virulence and the possibility of causing a streptococcal toxic shock-like syndrome (TSLS) vehiculated by superantigens [3]. In 1993, the Working Group on Severe Streptococcal Infection (WGSSI) formalized the criteria for GAS TSLS: a definite diagnosis made by GAS isolation in sterile media, signs, symptoms and typical laboratory abnormalities of septic shock; a probable diagnosis made by the latter and GAS isolation in a non-sterile site [4]. As its counterpart, GBS TSLS diagnosis is made similarly.

In severely burned patients, with the innate and adaptive

Manuscript submitted September 7, 2017, accepted September 18, 2017

aPlastic Surgery Department, Hospital de Santa Maria, Lisbon, Portugal ${ }^{b}$ Corresponding Author: Arielle Turpin, Plastic Surgery Department, Hospital de Santa Maria, Lisbon, Portugal. Email: arielleturpin@hotmail.com

doi: https://doi.org/10.14740/jmc2910w immunosuppression inherent to their condition, NF can rapidly progress to invasive systemic disease mimicking and adding to the burn's own pathophysiology. There are only 14 reported cases in Anglo-Saxon based literature of GBS TSLS, half of which starting from NF; none of them in a burn patient [3-7].

This article describes, to the author's knowledge, the first published case of TSLS by GBS in a severely burned patient starting from NF.

\section{Case Report}

A 23-year-old Caucasian male, firefighter, otherwise healthy was admitted to our institution evacuated from a wildfire that had been active for 1 week. He sustained $72 \%$ of total body surface area (TBSA), with deep burn distributed through trunk, four limbs and middle third of the face including palpebral and conjunctival burn. He also suffered upper airway burn and was intubated orotracheally on site. At arrival in our facilities he had completed the first $18 \mathrm{~h}$ of volemic reposition with lactate according to Parkland's formula. Despite hydration he was in shock with hypotension, hypothermia, oligoanuria, renal and liver laboratorial abnormalities and metabolic acidosis. He was also in acute respiratory distress syndrome (ARDS). We noted elevated compartment pressure on both lower limbs and left upper limb. Urine and blood cultures were made, skin, nasal and oropharynx swabs were taken and sputum harvested. He was rushed to theatre where fasciotomies of the referred limbs were performed (Fig. 1). There was leakage of pus from the right lower limb and a distinct necrotic appearance of its fascia. Samples of fascia and exudate were taken. Empiric amoxicillin plus clavulanic acid was started and changed 2 days later to ampicillin plus clindamycin once antibiogram was available, as Streptococcus agalactiae was isolated from right lower limb skin swab, exudate and fascia culture. Streptococcus pneumonia was also found in sputum culture with similar antibiotic sensitivity. All other cultures were negative. Once hemodynamic status allowed, at day 5 post-admission, right lower limb complete debridement and partial thickness skin grafting was made with great improvement of inflammatory parameters and general condition (Fig. 2). Debridement of contralateral lower limb, left upper limb, thorax, dorsum and right upper limb followed as hemodynamic stability allowed during the course of the next 20 days in a high to low affected area and life to limb threatening motto. A safety perimeter between grafted areas and non-debrided eschar was ensued. 


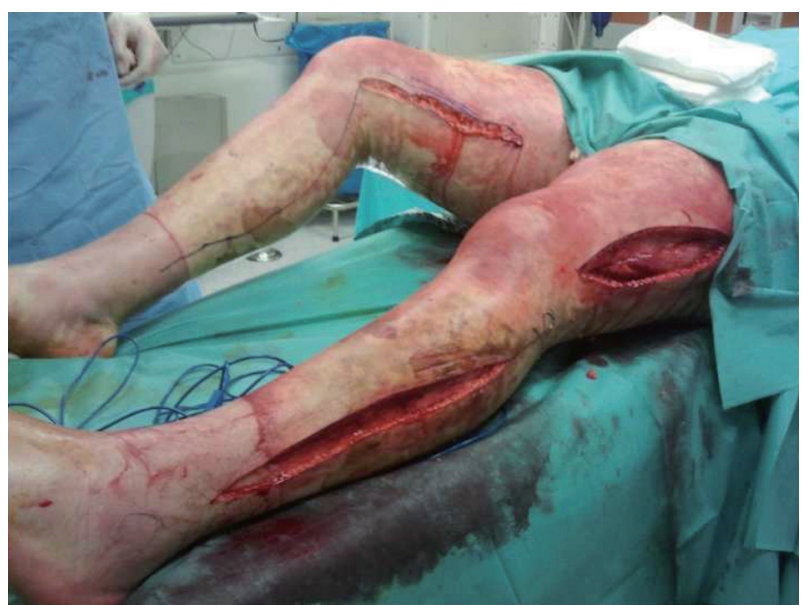

Figure 1. Fasciotomies of lower limbs at admission.

During that period of time there were some intervening complications to nosocomial and opportunistic flora including sparse focus of limb graft and undebrided eschar infection with Pseudomonas aeruginosa, Candida parapsilosis and Enterobacteriaceae. Tegument infection diminished as expected as debridement proceeded but there was need to re-graft some areas of both lower limbs and left upper limb during the course of the following month. Microbiological testing was done extensively and infections were treated accordingly. Aggressive physiotherapy started from hospitalization and continued after discharge. The patient was able to return to his previous professional activity totally healed with minimal impairment (Fig. $3)$.

\section{Discussion}

NF starting from burn lesions is a rare possibility. GBS is a rare NF pathogen and has been reported in only 22 cases in English based literature [3], in which none of them is a burn patient. In the particular case of burns, the acute loss of tegu-

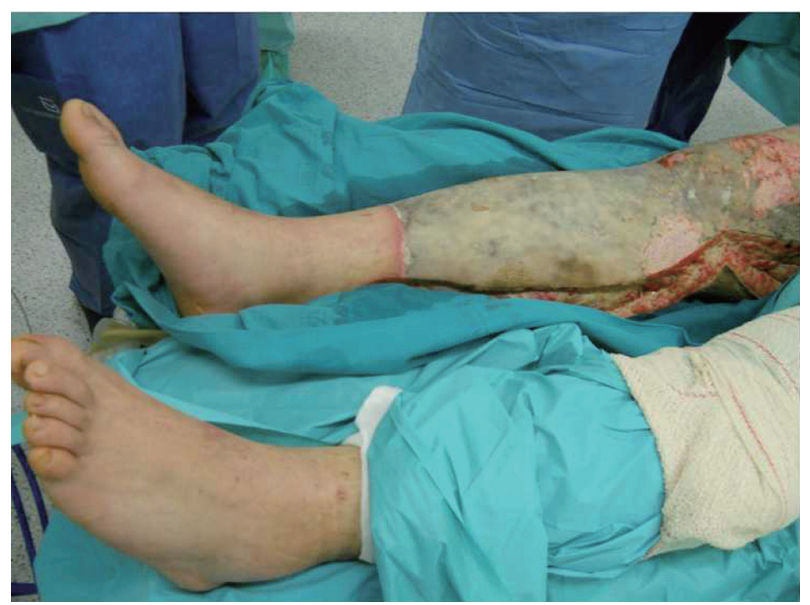

Figure 2. Right lower limb at day 5 post-admission before complete debridement.

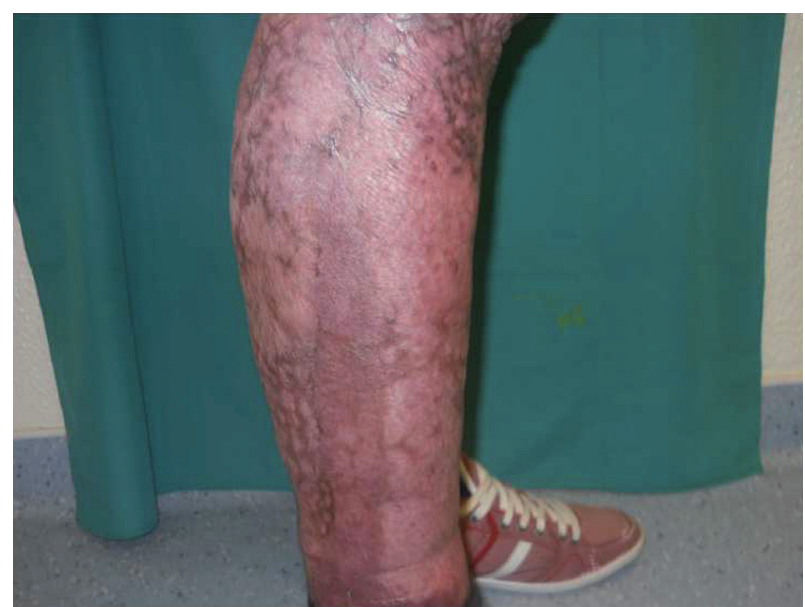

Figure 3. Right lower limb at 9 months post-operation completely healed.

ment leads the skin and mucosa commensals to deep planes, in a previously healthy host. Immunosuppression which also follows burns has a unique independent positive association with invasive GBS disease among non-pregnant adults that is consistent amongst multiple studies $[8,9]$. In our patient alluding to the GBS NF diagnosis we had the admission clinical picture with marked tissue edema, compartment syndrome, shock and multi-organ failure, the greyish necrotic deep fascia and pus leakage at wound exploration and finally GBS isolation in fascia and exudate along with skin swab. Of course it can be argued that the former signs were due to the systemic inflammatory response syndrome (SIRS) to the burn injury itself but the surgical, microbiological findings and clinical and laboratorial improvements with drainage and debridement leave no doubt that at the very least NF contributed to the initial clinical presentation alongside with the burn's own pathophysiology.

Another suspicion that can be raised at admission is if toxicosis from GBS superantigens could have also played a role, as WGSSI criteria were fulfilled for TSLS: isolation of GBS from a sterile media, hypotension with multi-organ failure including ARDS and NF [4]. Once again it could all be attributed to SIRS and NF, and the fact that GBS serotyping and toxin production tests were not made cannot thoroughly confirm diagnosis. Nevertheless the hypothesis cannot be ignored given all the favorable data and being so, our case is the first of its kind. In English literature there are only other 14 reported cases of GBS TSLS, half of which starting from NF and none of them in a burn patient [3-7]. Even if we consider the more frequent staphylococcal toxic shock syndrome (TSS) there are only two cases in adult patients with burns $[10,11]$.

TSS and by approximation TSLS in burns seems to be related to colonization of the burn wound with the pathogen (as was confirmed in our patient), environmental factors influencing toxin production, absence of protective antibodies (that by the second decade can ascend to $42 \%$ of the population as might have been the case of our patient) and genetic susceptibility to the pirotoxins (related to MHC loci and TCR type) [12].

Reviewing published data and in contrast to its staphy- 
lococcal counterpart which prevails in children (namely after burns), GBS TSLS seems to favor an older age and underlying immunosuppressive disease $[9,12]$. In our patient, massive loss of tegument from deep burn and subsequent immunosuppressive state might have been enablers of TSLS following NF.

As for therapeutic steps that were crucial for survival, given the likely conjoint action of major burn, NF and TSLS at admission, we had aggressive resuscitation support, prompt surgical debridement of infected tissue and adjusted antibiotherapy with clindamycin (that inhibits protein synthesis including toxins) and penicillin (that inhibits cell wall synthesis and prevents clindamycin resistance). Other measures with anecdotal evidence of effectiveness against superantigens production, like hyperbaric chamber and intravenous immunoglobulin, were not taken.

In conclusion, this singular combination of nosologic entities is the first of its kind and adds on to the list of what seems to be an emerging disease.

\section{Conflict of Interest}

The authors have no disclosures.

\section{Financial Support}

No funding was used to make this article.

\section{Ethical Approval}

This article was approved by the Ethical Council of Hospital Universitario de Santa Maria.

\section{References}

1. Golger A, Ching S, Goldsmith CH, Pennie RA, Bain JR. Mortality in patients with necrotizing fasciitis. Plast Re- constr Surg. 2007;119(6):1803-1807.

2. Wong CH, Kurup A, Tan KC. Group B Streptococcus necrotizing fasciitis: an emerging disease? Eur J Clin Microbiol Infect Dis. 2004;23(7):573-575.

3. Fukuda K, Ryujin M, Sakio R, Fukuzumi S, Omae T, Hayakawa K. Bilateral necrotizing fasciitis of the foot associated with Group B streptococcus. Case Rep Dermatol. 2016;8(3):243-249.

4. Holmstrom B, Grimsley EW. Necrotizing fasciitis and toxic shock-like syndrome caused by group B streptococcus. South Med J. 2000;93(11):1096-1098.

5. Crum NF, Wallace MR. Group B streptococcal necrotizing fasciitis and toxic shock-like syndrome: a case report and review of the literature. Scand J Infect Dis. 2003;35(11-12):878-881.

6. Tang WM, Ho PL, Yau WP, Wong JW, Yip DK. Report of 2 fatal cases of adult necrotizing fasciitis and toxic shock syndrome caused by Streptococcus agalactiae. Clin Infect Dis. 2000;31(4):E15-17.

7. Gardam MA, Low DE, Saginur R, Miller MA. Group $\mathrm{B}$ streptococcal necrotizing fasciitis and streptococcal toxic shock-like syndrome in adults. Arch Intern Med. 1998;158(15):1704-1708.

8. Skoff TH, Farley MM, Petit S, Craig AS, Schaffner W, Gershman K, Harrison LH, et al. Increasing burden of invasive group B streptococcal disease in nonpregnant adults, 1990-2007. Clin Infect Dis. 2009;49(1):85-92.

9. Sunkara B, Bheemreddy S, Lorber B, Lephart PR, Hayakawa K, Sobel JD, Kaye KS, et al. Group B Streptococcus infections in non-pregnant adults: the role of immunosuppression. Int J Infect Dis. 2012;16(3):e182-186.

10. Withey SJ, Carver N, Frame JD, Walker CC. Toxic shock syndrome in adult burns. Burns. 1999;25(7):659-662.

11. Jarman A, Duggal A, Korus L, Tredget EE. Toxic shock syndrome in an adult burn patient. Burns. 2007;33(8):1051-1053.

12. Al Akhrass F, Abdallah L, Berger S, Hanna R, Reynolds N, Thompson S, Hallit R, et al. Streptococcus agalactiae toxic shock-like syndrome: two case reports and review of the literature. Medicine (Baltimore). 2013;92(1):10-14. 